\title{
MGMT-Independent Temozolomide Resistance in Pediatric Glioblastoma Cells Associated with a PI3-Kinase-Mediated HOX/Stem Cell Gene Signature
}

\author{
Nathalie Gaspar ${ }^{1,2,3}$, Lynley Marshall ${ }^{1,2,4}$, Lara Perryman ${ }^{1}$, Dorine A. Bax ${ }^{1}$, Suzanne E. Little ${ }^{1}$, \\ Marta Viana-Pereira ${ }^{1,5}$, Swee Y. Sharp ${ }^{2}$, Gilles Vassal ${ }^{4}$, Andrew D.J. Pearson ${ }^{1,3}$, \\ Rui M. Reis ${ }^{5}$, Darren Hargrave ${ }^{3}$, Paul Workman ${ }^{2}$, and Chris Jones ${ }^{1}$
}

Abstract

Sensitivity to temozolomide is restricted to a subset of glioblastoma patients, with the major determinant of resistance being a lack of promoter methylation of the gene encoding the repair protein DNA methyltransferase MGMT, although other mechanisms are thought to be active. There are, however, limited preclinical data in model systems derived from pediatric glioma patients. We screened a series of cell lines for temozolomide efficacy in vitro, and investigated the differential mechanisms of resistance involved. In the majority of cell lines, a lack of MGMT promoter methylation and subsequent protein overexpression were linked to temozolomide resistance. An exception was the pediatric glioblastoma line KNS42. Expression profiling data revealed a coordinated upregulation of $H O X$ gene expression in resistant lines, especially KNS42, which was reversed by phosphoinositide 3-kinase pathway inhibition. High levels of HOXA9/HOXA10 gene expression were associated with a shorter survival in pediatric high-grade glioma patient samples. Combination treatment in vitro of pathway inhibition and temozolomide resulted in a highly synergistic interaction in KNS42 cells. The resistance gene signature further included contiguous genes within the 12q13-q14 amplicon, including the Akt enhancer PIKE, significantly overexpressed in the KNS42 line. These cells were also highly enriched for CD133 and other stem cell markers. We have thus shown an in vitro link between phosphoinositide 3-kinase-mediated HOXA9/HOXA10 expression, and a drug-resistant, progenitor cell phenotype in MGMT-independent pediatric glioblastoma. Cancer Res; 70(22); 9243-52. (2010 AACR.

\section{Introduction}

Glioblastoma is the most common tumor of the central nervous system, affecting patients of all ages, and being essentially refractory to treatment; the clinical outcome remains dismal regardless of age at diagnosis. The median survival of a patient with glioblastoma is 15 months, and this has improved little in the last four decades (1). The mainstays of treatment during this time have been surgical resection and radiotherapy, often with nitrosurea-based chemotherapy. The use of adjuvant temozolomide has more recently emerged as a new standard of care in glioblastoma, with concurrent and sequential treatment in the initial therapy of patients resulting in a modest improvement in median survival (2).

Authors' Affiliations: ${ }^{1}$ Paediatric Oncology, and ${ }^{2}$ Cancer Research UK Centre for Cancer Therapeutics, The Institute of Cancer Research, and ${ }^{3}$ Paediatric Oncology, The Royal Marsden NHS Foundation Trust, Sutton, United Kingdom; ${ }^{4}$ Pharmacology and New Treatments of Cancer, Institut de Cancérologie Gustav Roussy, Villejuif, France; and ${ }^{5}$ Life and Health Science Research Institute (ICVS), Universidade do Minho, Braga, Portugal

Corresponding Author: Chris Jones, Paediatric Oncology, The Institute of Cancer Research, 15 Cotswold Road, Sutton, Surrey, SM2 5NG, United Kingdom. Phone: 44-0-20-8722-4416; Fax: 44-0-20-8722-4321; E-mail: chris.jones@icr.ac.uk.

doi: 10.1158/0008-5472.CAN-10-1250

(C)2010 American Association for Cancer Research.
Temozolomide is a DNA methylating agent that is orally bioavailable, crosses the blood:brain barrier, and exhibits schedule-dependent antitumor activity (3). The improved survival benefits in glioblastoma are largely restricted to the subset of patients lacking expression of the DNA repair enzyme $\mathrm{O}^{6}$-methylguanine-DNA-methyl-transferase (MGMT; ref. 4). Temozolomide induces cytotoxic $\mathrm{O}^{6}$-guanine methyl adducts that are removed directly by functional MGMT, thereby producing drug resistance. Downregulation of MGMT usually occurs in tumors by gene promoter hypermethylation, in which $>50 \%$ methylation has been shown to silence gene expression (5).

Deficiencies in DNA mismatch repair (MMR) are also linked to resistance to alkylating agents such as temozolomide (6), as are elevated levels of Ape1/Ref-1, a major component of the base excision repair (BER; ref. 7) system, with attempts to enhance temozolomide-induced cytotoxicity by disrupting BER by means of inhibition of poly-(ADP-ribose)-polymerase (PARP) proving effective in vitro and in vivo (8).

The vast majority of the above work has taken place in adult glioblastoma and preclinical models derived from adult patients. In the pediatric setting, MGMT promoter hypermethylation predicts for response to alkylating agents (9); however, the survival of children treated with adjuvant temozolomide does not seem to be improved when compared with historical controls (10-14). The mechanisms of drug resistance in 
pediatric high-grade glioma are poorly understood, in part due to the lack of availability of suitable models of the disease. We screened a series of pediatric and adult glioma cell lines for temozolomide efficacy in vitro, and investigated the differential mechanisms of resistance involved, highlighting the involvement in pediatric cells of processes outside of the usual MGMT/MMR/BER axis.

\section{Materials and Methods}

\section{Cell culture}

Adult glioblastoma cell lines A172, LN229, SF268, U87MG, U118MG, and U138MG, and pediatric glioma cell lines SF188, KNS42, UW479, Res259, and Res186 were obtained and cul- tured as previously described (15). For the spheroid formation assay, cells were grown in neurosphere medium, which consisted of NDiff RHB-A medium (Stem Cell Sciences) supplemented with epidermal growth factor and fibroblast growth factor 2 , each at $20 \mathrm{ng} / \mathrm{mL}$.

\section{Growth inhibition studies}

Temozolomide was obtained from Apin Chemicals, $\mathrm{O}^{6}-$ benzylguanine from Calbiochem, and PI-103 from Piramed Pharma or synthesized in-house. Growth inhibition was determined using the sulforhodamine B (16) or MTS (17) assay as previously described. To attempt reversion of resistance to temozolomide, $\mathrm{O}^{6}$-benzylguanine was added at the highest nontoxic concentration (10-15\% of cell growth inhibition,

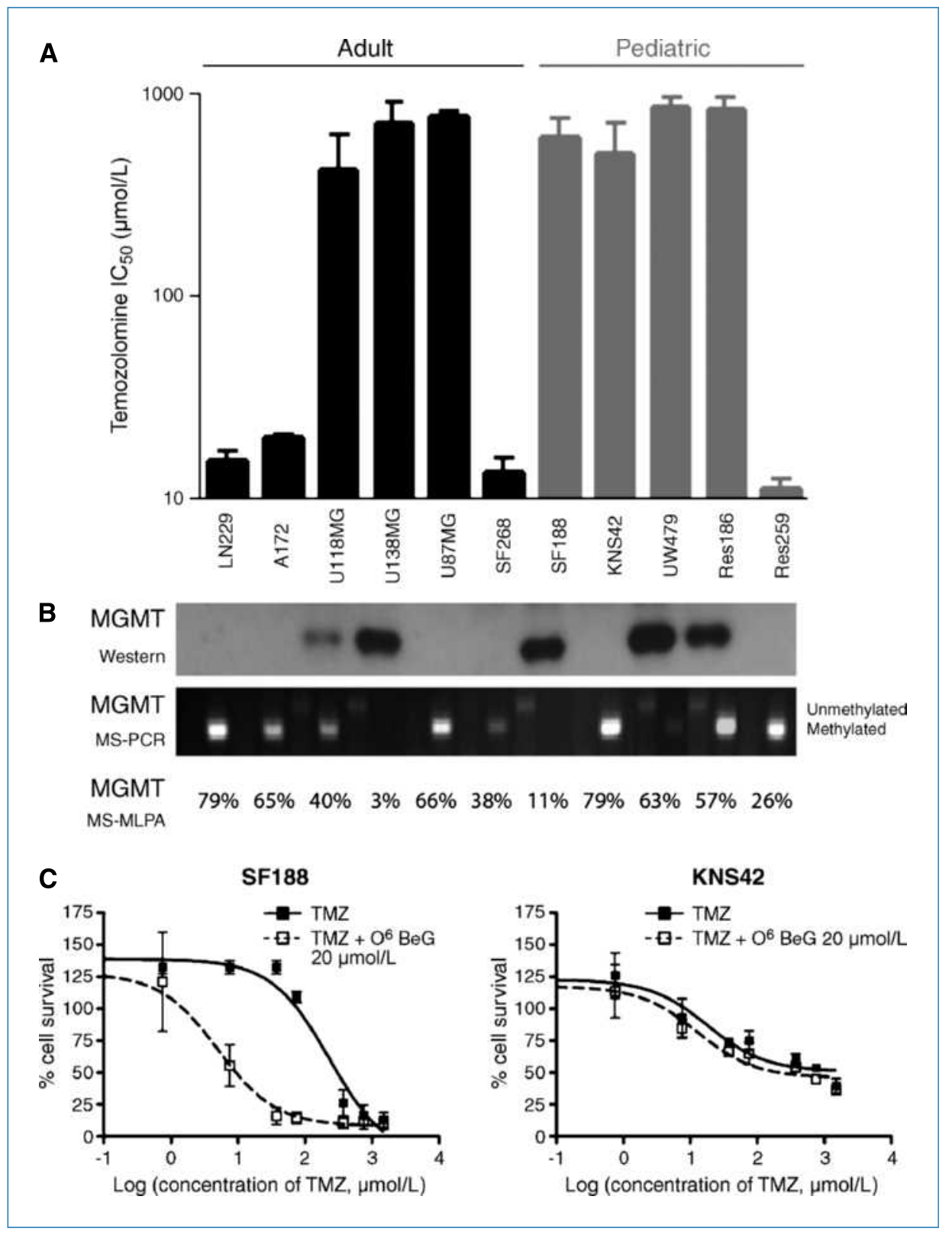

Figure 1. Sensitivity of pediatric and adult glioma cell lines to temozolomide and relationship to MGMT status. A, adult (LN229, A172, U118MG, U138MG, U87MG, SF268) and pediatric (SF188, KNS42, UW479, Res186, Res259) glioma cells were treated with temozolomide, and cytotoxicity was assessed by the sulforhodamine $B$ assay. $\mathrm{IC}_{50}$ values are plotted on a $\log _{10}$ scale. B, Western blot for MGMT protein expression correlated with extent of promoter methylation as assessed by MS-PCR and MS-MLPA. In most cases expression correlates with temozolomide resistance, with the exception of U87MG and KNS42 cells, which are hypermethylated, do not express the protein, and are resistant to temozolomide. C, SF188 and KNS42 cells were treated with MGMT substrate analogue $\mathrm{O}^{6}$-benzylguanine, showing the MGMT-dependent nature of temozolomide (TMZ) resistance in SF188, but not KNS42 cells. Growth inhibition was determined by the sulforhodamine $\mathrm{B}$ assay. Concentration of temozolomide is on a $\log _{10}$ scale. 


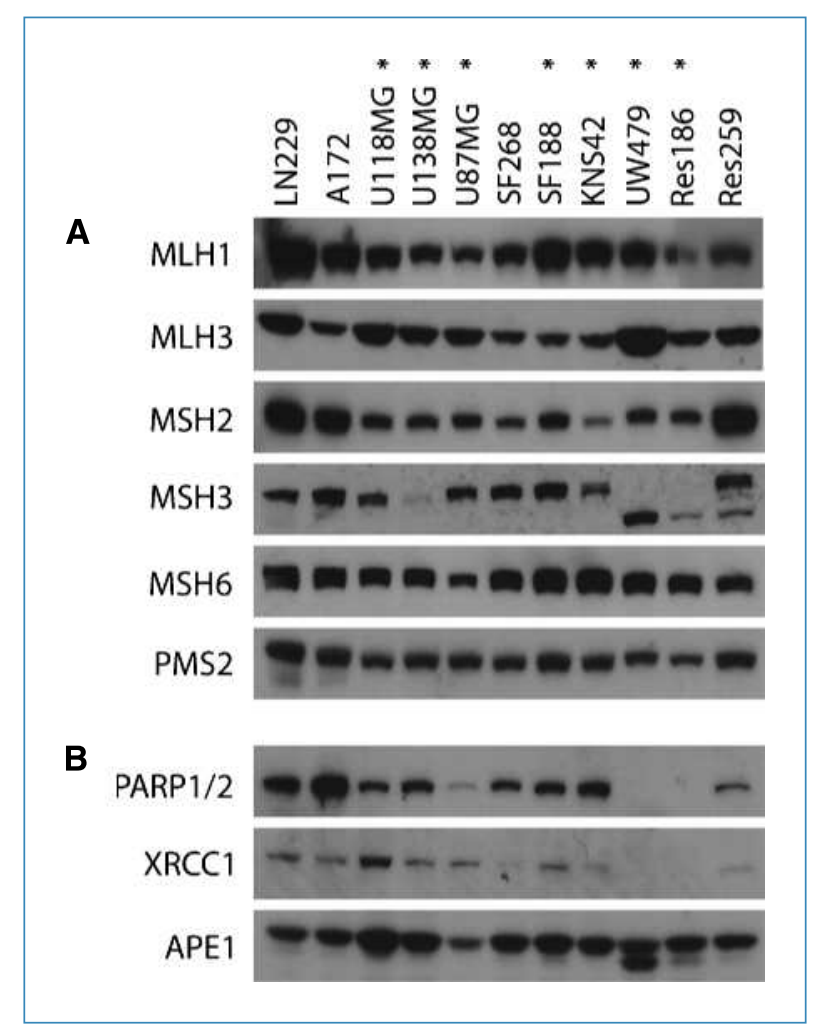

Figure 2. Assessment of DNA MMR and BER pathways in pediatric and adult glioma cell lines. Western blot for proteins involved in MMR (A) and BER (B). Although deficiencies in MSH3 are noted in U138MG, UW479, and Res186, and there is some variability in PARP and XRCC1 expression, U87MG and KNS42 have normal expression of proteins involved in both pathways. *, temozolomide-resistant cell lines.

$20 \mu \mathrm{mol} / \mathrm{L}$ ). For the assessment of combination effects, cells were treated with increasing concentrations of drugs either alone or concurrently at their equipotent molar ratio and combination indices were calculated by the method of Chou and Talalay (18). All values are given as mean \pm SD of at least three independent experiments.

\section{Promoter methylation analysis}

Cell line DNA was treated with sodium bisulphite using the Epitect kit (Qiagen) according to the manufacturer's instructions. Methylation-specific (MS) PCR for the MGMT promoter was performed as described previously (19). MS-multiplex ligation-dependent probe amplification (MLPA) was carried out as previously reported (15) according to the manufacturer's instructions (MRC-Holland; ref. 20). HOXA9/HOXA10 methylation was assessed by comparing expression profiles of 5-Aza-2'-deoxycytidine-treated cells with vehicle-treated controls on Illumina Human-6 v2 Expression BeadChips (Illumina Inc.), ArrayExpress accession number E-TABM-858.

\section{Western blot analysis}

Immunodetection was performed as previously described (15) using antibodies against MGMT (1:500; Zymed), MLH1 (1:500; Pharmingen), MLH3 (1:500; Santa Cruz Biotechnolo- gies), MSH2 (1:500; Calbiochem), MSH3 (1:250; BD Bioscience), MSH6, PMS2 (both 1:500; BD Bioscience), PARP1/2 (1:1,000; Cell Signaling), XRCC1 (1:500; Cell Signaling), APE1 (Novus Biochemicals), p85, p110 $\alpha$ (Cell Signaling), p110 $\beta$, p110א (Santa Cruz), PIKE-A/PIKE-L (all 1:1,000; Abcam), phospho-Akt ${ }^{\text {Ser473}}$, Akt (both 1:1,000; Cell Signaling), and GAPDH (1:2,000; Chemicon).

\section{mRNA expression profiling analysis}

Cell line expression profiling by Affymetrix U133 oligonucleotide arrays has been previously described (ref. 15; ArrayExpress accession number E-TABM-579). Supervised analysis was performed using an absolute signal-to-noise metric of $>1.5$ in GenePattern software (http://www.broad.mit.edu/cancer/ software/genepattern/). Coordinate gene regulation was identified using gene set enrichment analysis (GSEA; www.broad. mit.edu/gsea/), with a nominal $P$ value cutoff of 0.001 . "Core enriched" genes are defined as belonging to the leading-edge subset within the gene set, and thus contribute the most to the enrichment result. Assessment of $\mathrm{HOX}$ gene expression after 24-hour treatment with PI-103 at $5 \times \mathrm{IC}_{50}$ was carried out using Illumina HT-12 BeadChips (ArrayExpress accession number E-TABM-890). Affymetrix U133 expression data from The Cancer Genome Atlas (TCGA) glioblastoma study (21) was assessed for cross-correlations of probesets corresponding to HOXA9 by calculating Pearson's correlation coefficients in R. GSEA and clinical correlations were further carried out on a published dataset (22) of Affymetrix U133 expression array profiling of 78 pediatric high-grade gliomas (Gene Expression Omnibus accession number GSE19578; http://www.ncbi.nlm.nih.gov/geo/).

\section{Immunofluorescence and flow cytometry}

CD133 protein expression was measured by both flow cytometry using a BD fluorescence-activated cell sorting Vantage SEDiVa system (BD Biosciences) and immunofluorescence on cytospin preparations, using anti-CD133 antibody (AC133/1, Miltenyi Biotec) at 1:50 and 1:100 dilution, respectively. Cells were costained with nestin (196908, R \& D Systems) and visualized with 4', 6-diamidino-2-phenylindole (DAPI; Vector Laboratories Inc.). Cell cycle analysis was also carried out by flow cytometry.

\section{Results}

\section{Sensitivity of glioma cell lines to temozolomide in vitro is largely but not exclusively dependent on MGMT promoter methylation and lack of protein expression}

We first determined the response to temozolomide in vitro of our panel of five pediatric (SF188, KNS42, UW479, Res259, Res186) and six adult (A172, LN229, SF268, U87MG, U118MG, U138MG) glioma cell lines. Four lines (A172, LN229, SF268, Res259) were classed as temozolomide sensitive, with $\mathrm{IC}_{50}$ values of between 10 and $20 \mu \mathrm{mol} / \mathrm{L}$ (Fig. 1A). The remaining cells were resistant to treatment with the alkylating agent, with $\mathrm{IC}_{50}$ values of $>500 \mu \mathrm{mol} / \mathrm{L}$.

We assessed MGMT promoter methylation by methylationspecific PCR and MLPA, and protein expression by Western 


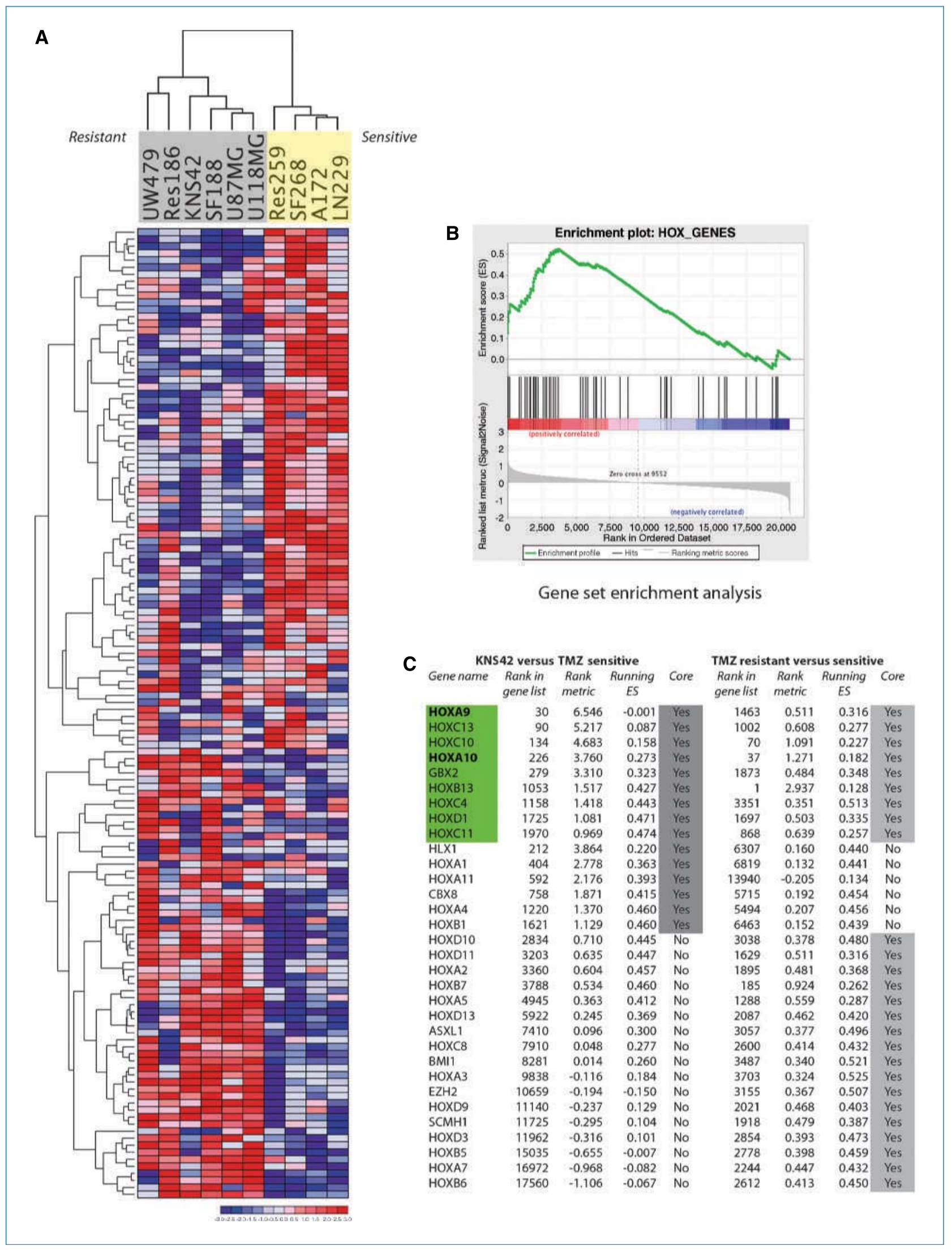


blot (Fig. 1B). Extensive methylation resulted in an absence of MGMT protein expression in LN229, A172, U87MG, SF268, Res259, and KNS42 cells. There was, for the most part, a direct correlation between MGMT methylation/lack of expression and temozolomide sensitivity. An exception to this was the pediatric glioblastoma KNS42 cell line, which displayed insensitivity in the absence of MGMT protein, implying that alternate mechanisms of resistance must be operative. To confirm this, we treated pediatric glioblastoma SF188 and KNS42 cells with temozolomide in the presence of the substrate analogue $\mathrm{O}^{6}$-benzyl guanine $\left(\mathrm{O}^{6} \mathrm{BeG}\right)$, which depletes the enzyme and increases cytotoxicity (ref. 23; Fig. 1C). Treatment with $20 \mu \mathrm{mol} / \mathrm{L} \mathrm{O}^{6} \mathrm{BeG}$ increased the efficacy of temozolomide in SF 188 cells nearly 40 -fold ( $\mathrm{IC}_{50}$ without $\mathrm{O}^{6} \mathrm{BeG}=194 \mu \mathrm{mol} / \mathrm{L}$; $\mathrm{IC}_{50}$ in the presence of $\mathrm{O}^{6} \mathrm{BeG}=5 \mu \mathrm{mol} / \mathrm{L}$ ), thus confirming the dependence of these cells on MGMT in conferring temozolomide resistance. By contrast, no such effect was seen in KNS42 cells, showing the MGMT-independent nature of the insensitivity.

\section{Dysregulation of MMR and BER proteins do not explain resistance to temozolomide in MGMT-deficient KNS42 cells}

Enzymes involved in DNA MMR (MLH1, MLH3, MSH2, MSH3, MSH6, PMS2) were evaluated by Western blot (Fig. 2A). Although we identified three temozolomideresistant lines with abrogated expression of MSH3 (U138MG, UW479, and Res186), there was no deficiency in KNS42 or U87MG cells. These data correlated well with levels of promoter methylation assessed by MS-MLPA(15). We further investigated components of the BER pathway, including PARP1/2, XRCC1, and APE1. Although temozolomide-resistant UW479 and Res186 seemed to lack PARP1/2 and XRCC1 expression, there were no apparent alterations in other glioma lines, including KNS42 or U87MG (Fig. 2B).

\section{Identification of a $\mathrm{HOX} /$ stem cell gene expression signature associated with temozolomide resistance in pediatric glioblastoma cells}

Using expression microarrays, we identified 135 genes differentially expressed between sensitive and resistant glioma cell lines (Fig. 3A). Included in this list were MGMT and PARP2, despite these enzymes not explaining the resistance in all cell lines. Also included were several kinases, including MAPK9 and $C D K 6$, which may prove suitable targets for pharmacologic modulation; PIK3C3 (Vps34), suggesting a possible link to the autophagic response (24); and genes encoding elements of the immune response such as IL10 and IL16.
When we applied GSEA to our data, we observed coordinated differential expression of the HOX_GENES set (MSigDB C2:curated gene sets) in resistant versus sensitive cell lines (Fig. 3B), with an enrichment score of 0.54 [nominal $P=0.01$; false discovery rate (FDR) $q=0.403$ ]. Furthermore, the HOX_GENES list was also identified as significant using a GSEA "Preranked" analysis based on differentially expressed genes between KNS42 alone and temozolomide-sensitive cell lines (Fig. 3C). In these analyses, the genes in both coreenriched lists, which contribute to the leading-edge subset within the gene set (25), included HOXA9, HOXA10, HOXB13, HOXC4, HOXC10, HOXC11, HOXC13, HOXD1, and GBX2.

As coordinated expression of $H O X$ genes had recently been noted in glioblastoma clinical samples (26), and was reported as evidence of a "self-renewal" signature as it included the stem cell marker PROM1 (CD133), we sought further evidence for this in a published glioblastoma dataset and our cell line models. When we investigated TCGA expression profiles of 163 glioblastomas for genes that correlated with the top-ranking $H O X$ gene in our KNS42 GSEA list, $H O X A 9$, we noted a remarkable parallel expression of numerous other homeobox genes (Fig. 4A). Of the top 47 genes by this analysis, 18 were homeobox genes found at 9 distinct genomic loci, and 7 were included in the self-renewal signature of Murat and colleagues (26). These latter genes included PROM1. Intriguingly, the vast majority of the non-homeobox genes identified by this analysis are contiguous genes found commonly amplified in glioblastoma at the genomic locus 12q13-q14.

To determine whether an enrichment of the stem cell marker CD133 may be playing a role in the resistance of KNS42 cells to temozolomide, we assessed the levels of mRNA expression relative to the other cell lines (Fig. 4B), and noted considerably higher levels of PROM1 in KNS42 cells than in any other line in our panel. Of note, the only other two cell lines to express $P R O M 1$ at above background levels were the similarly temozolomide-resistant U87MG and SF188. This was visualized by immunofluorescent staining for CD133, colabeled with nestin (Fig. 4C). We had previously reported the relatively high levels of stem cell markers in SF188 and KNS42 by immunocytochemistry (15), and to more accurately quantify this, we used the more sensitive flow cytometry analysis to reveal an usually high degree of expression in KNS42, with $17.0 \%$ of cells positive for CD133. There were also high levels of CD133-positive cells in the SF 188 line (4.8\%) compared with $0.0 \%$ to $0.02 \%$ in other cell lines. KNS42 cells grown as monolayers also expressed by far the greatest levels of other stem cell markers such as nestin, SOX2, and musashi-1 (Affymetrix U133, data

Figure 3. Expression profiling reveals a HOX/stem cell signature-associated temozolomide resistance in glioma cell lines. A, heatmap showing hierarchical clustering of 135 differentially expressed genes between resistant (UW479, Res186, KNS42, SF188, U87MG, U118MG; gray highlight) and sensitive (Res259, SF268, A172, LN229; yellow highlight) high-grade glioma cell lines. B, GSEA highlighting coordinated differential expression of gene sets defined a priori. Enriched in temozolomide-resistant cell lines were the HOX_GENES set (enrichment score $=0.54$, nominal $P=0.01$; FDR $q=0.403$ ). C, ranked genes derived from GSEA of KNS42 versus temozolomide-sensitive cell lines, and all temozolomide-resistant versus sensitive lines. Genes are provided along with their rank in the total gene list and rank enrichment metric, and running enrichment scores are provided for both analyses. Gray highlight, genes present within the core enrichment signature; green highlight, genes present in both analyses; bold, genes also present in the $H O X$ gene signature in Murat and colleagues (26), HOXA9 and HOXA10. 
not shown). Consistent with the cancer stem/progenitor cellassociated gene expression profile, neurosphere formation assays showed that KNS42 cells form tight three-dimensional spheroids, which may be serially passaged and undergo selfrenewal. In contrast, SF188 cells form smaller, more loosely packed spheres, whereas U87MG grow as cell aggregates rather than neurospheres per se. Neither UW479, Res259, nor Res186 formed spheres under these conditions. Taken together, KNS42 cells seem to have a significant cancer stem/progenitor cellassociated gene expression signature and biological phenotype.

\section{Expression of $\mathrm{HOXA9} / \mathrm{HOXA10}$ is a result of phosphoinositide 3-kinase-mediated demethylation, inhibition of which synergistically interacts with temozolomide in KNS42 cells}

Treatment with the demethylating agent 5-aza-2'-deoxycytidine resulted in highly differential levels of expression of $H O X A 9$ and HOXA10 in all pediatric cell lines with the exception of KNS42, in which no changes were observed, indicative of a lack of methylation in the untreated cells (Fig. 5A). As a recent study has proposed a mechanism for this observation whereby transcriptional activation of the HOXA cluster is reversible by a phosphoinositide 3-kinase (PI3K) inhibitor through an epigenetic mechanism involving histone H3K27 trimethylation (27), we sought to investigate whether this mechanism was active in our system. Treatment for 1,8 , and 24 hours with the dual PI3K/mTOR inhibitor PI-103 (28-30) at $5 \times \mathrm{IC}_{50}$ resulted in significantly reduced expression of both HOXA9 and $H O X A 10$ in KNS42 cells in a time-dependent manner (Fig. 5B). These effects were not due to fluctuations in $H O X$ gene expression with the cell cycle, as diminished HOXA9/HOXA1O was observed as early as one hour posttreatment, at which time there was no evidence of $\mathrm{G}_{1}$ arrest (Fig. 5C), despite inhibition of PI3K as seen by reduced phospho-Akt levels (Fig. 5D).

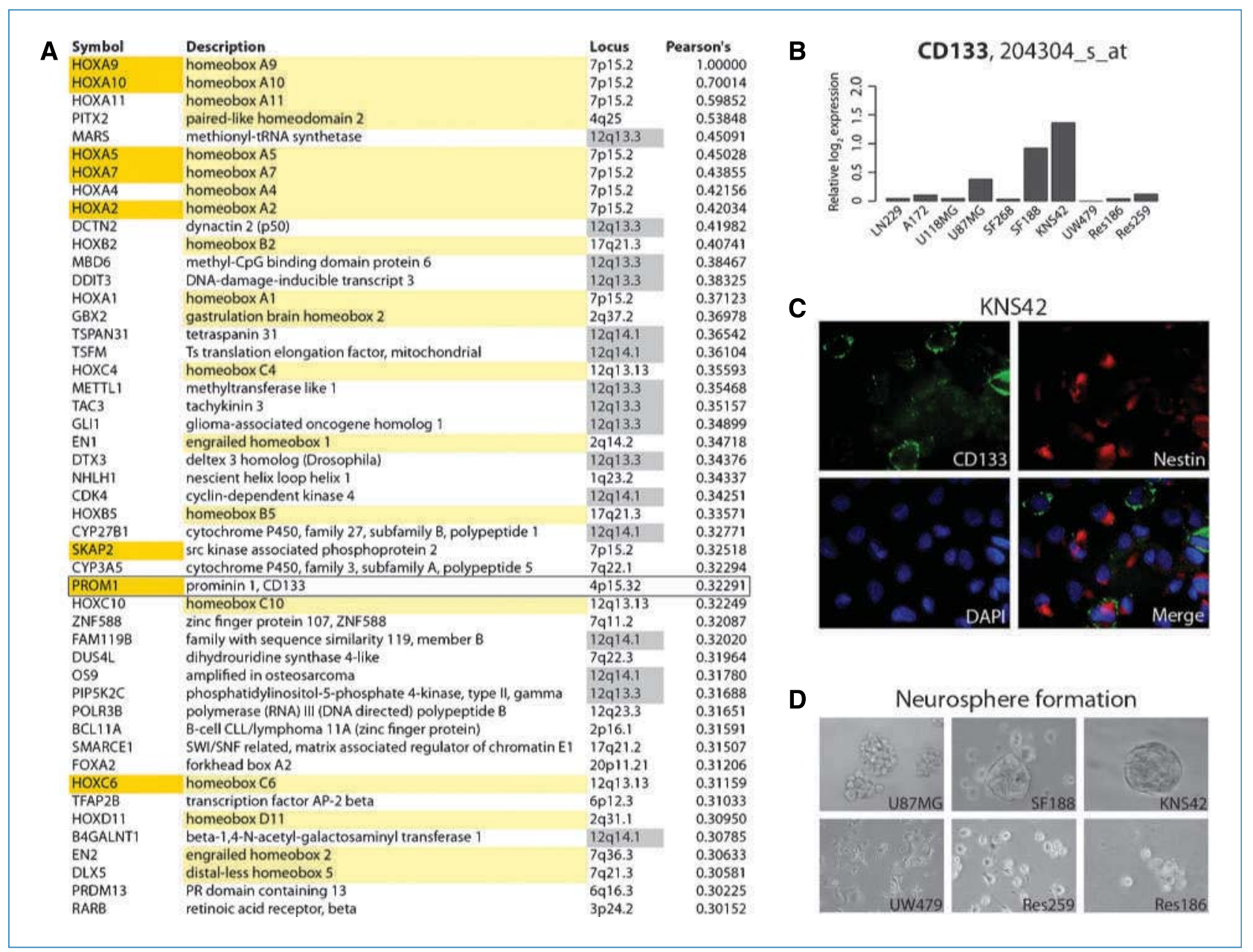

Figure 4. Coordinated upregulation of HOX genes in primary glioblastomas and a striking degree of CD133 positivity on KNS42 cells. A, genes coordinately expressed with HOXA9 from the TCGA dataset (21) were determined by calculating Pearson's correlation coefficients. All genes with values $>0.3$ are listed, in rank order. Yellow highlight, homeobox genes; orange highlight, genes also present in the HOX gene/self-renewal signature of Murat and colleagues (26), including PROM1 (CD133; box); gray highlight, genes found within the 12q13-q14 amplicon. B, Affymetrix expression analysis of PROM1 (CD133) mRNA levels in the panel of glioma cell lines, plotted as relative $\log _{2}$ expression. C, immunofluorescence assay showing extensive expression of stem cell markers CD133 (green) and nestin (red) in KNS42 cells grown as a monolayer. D, neurosphere formation assay highlighting the tight three-dimensional spheroids formed by KNS42 cells in contrast to the other cell lines studied. 


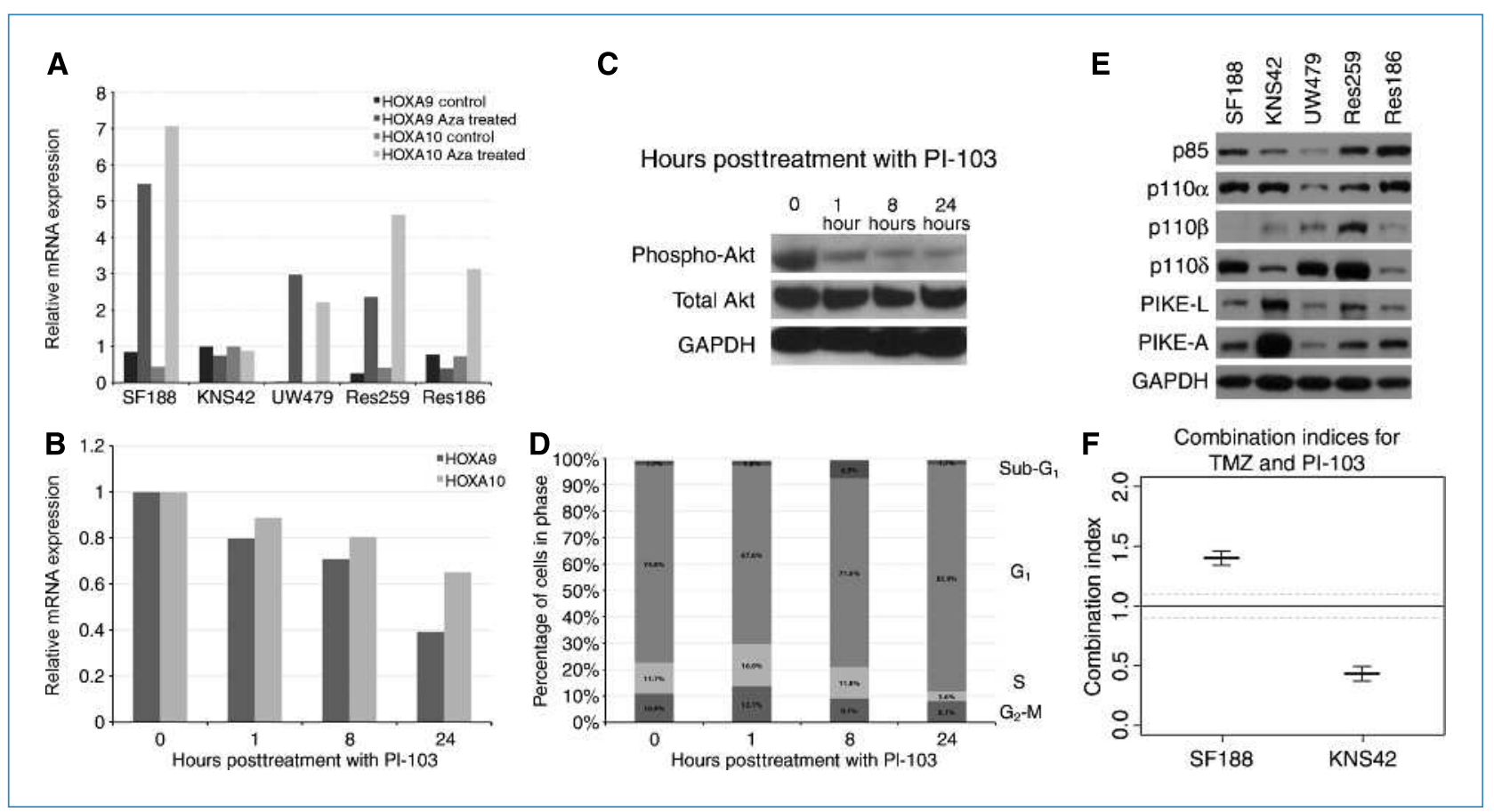

Figure 5. HOXA9/HOXA10 expression in KNS42 cells is driven by a lack of promoter methylation in a PI3K-dependent manner. A, relative mRNA expression levels of HOXA9 and HOXA10 before and after treatment with 5-Aza-2'-deoxycytidine in pediatric glioma cell lines. An absence of expression changes after 5-Aza-2'-deoxycytidine treatment in KNS42 cells is indicative of an absence of constitutive promoter methylation. B, HOXA9/HOXA10 expression is reduced by treatment with PI-103 in KNS42 cells in a time-dependent manner. Treatment with the dual $\mathrm{PI} 3 \mathrm{~K} / \mathrm{mTOR}$ inhibitor $\mathrm{PI}-103$ at $5 \times \mathrm{IC}_{50}$ for 1,8 , and 24 hours led to a reduction in expression of both HOXA9 and HOXA10 in KNS42 cells as early as 1 hour posttreatment. C, Western blot analysis of phospho- and total Akt after treatment of KNS42 cells with PI-103 at $5 \times \mathrm{IC}_{50}$ for 1,8 , and 24 hours. Inhibition of PI3K signaling is observed at the earliest time point. D, cell cycle analysis of KNS42 cells after treatment with $\mathrm{PI}-103$ at $5 \times \mathrm{IC}_{50}$ for 1,8 , and 24 hours. There was no $\mathrm{G}_{1}$ arrest evident at the early time points at which reduced $H O X A 9 /$ HOXA10 expression was observed. E, Western blot analysis of PI3K regulatory and catalytic subunits and enhancers in pediatric glioma cells. Expression of the specific Akt enhancer proteins PIKE-A and PIKE-L were considerably elevated in KNS42 cells compared with other pediatric glioma cells. F, synergistic interaction of $\mathrm{PI}-103$ and temozolomide (TMZ). Cotreatment with PI-103 and temozolomide resulted in a high degree of synergy in MGMT-independent KNS42 cells (combination index $=0.43$ ) as calculated by the median effect analysis. By contrast, SF188 cells showed an antagonistic interaction (combination index $=1.401$ ).

Next we sought to determine whether there was any specific dysregulation of the PI3K/PTEN system in KNS42 cells that may be responsible for the $H O X$ gene overexpression. Mutation screening for PTEN, PIK3CA, PIK3R1, and PIK3R3 did not identify any sequence variations (data not shown), and Western blot analysis confirmed a lack of overexpression of PI3K regulatory and catalytic subunits (Fig. 5E). By contrast, there were significantly elevated levels of the enhancer proteins PIKE-A and especially PIKE-L in KNS42 cells in comparison with the other lines. Both PIKE proteins are encoded by the CENTG1 (AGAP2) gene found within the 12q13q14 amplicon coordinately upregulated in association with the $H O X$ cluster, and likely represent a significant target for this genomic event in human glioblastoma.

Finally, we investigated the efficacy of targeting PI3K as a strategy for overcoming temozolomide resistance in our pediatric glioma cells. Combination treatment in vitro of temozolomide with the dual PI3K/mTOR inhibitor PI-103 resulted in a highly synergistic interaction in KNS42 as measured by median effect analysis (combination index $=0.43$; Fig. 5F). By contrast, in SF188 cells an antagonistic response was observed with the same combination (combination index $=1.40$ ).

\section{HOXA9/HOXA10 expression is associated with shorter survival in pediatric high-grade glioma patients}

To assess the translational relevance of $H O X$ gene expression in pediatric high-grade glioma patient samples, we examined published data detailing expression profiles of 78 tumors arising in childhood (22). Although the number of long-term ( $>3$ years) survivors is small, we identified 49 genes that were differentially expressed between patients with long and short ( $<1$ year) overall survival (Fig. 6A). Included in this list were $H O X A 2, H O X A 5, H O X 7$, and $H O X A 9$. By applying GSEA to the dataset, we identified coordinated upregulation in the short-term survivors of genes at the chromosome $7 \mathrm{p} 15$ cytoband, with an enrichment score of 0.68 (nominal $P<0.001$ ) albeit with a high false discovery rate value (FDR $q=0.930$ ). With the HOXA cluster found at this locus, running GSEA on the $H O X A$ gene list itself gave a highly significant enrichment score of 0.90 (nominal $P<0.001$, FDR $q<0.001$; Fig. 6B). There was considerable correlation between gene expression of all members of the HOXA family in the pediatric samples (Pearson's correlation coefficients 0.03-0.90). Taking the values of HOXA9 and HOXA10, and segregating samples into "high" and "low" expressers (combined expression greater or less than the 75 th percentile of all values, respectively), we showed a 


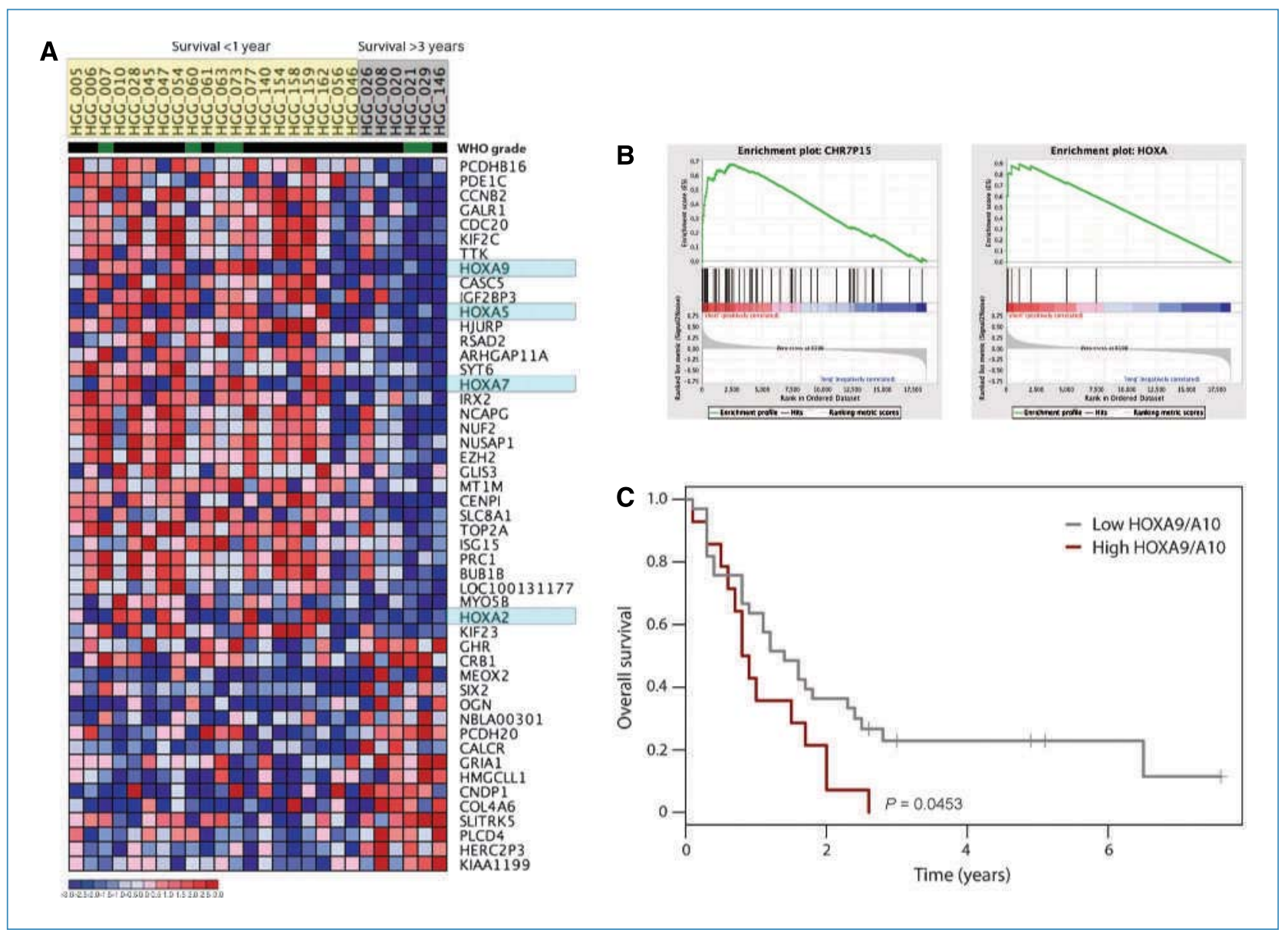

Figure 6. High levels of HOXA gene expression are associated with shorter survival in pediatric high-grade glioma patients. A, heatmap representing differentially expressed genes between short ( $<1$ year; yellow highlight) and long-term ( $>3$ years; gray highlight) survivors. WHO grade IV (black) and III (green) tumors are indicated. Light blue, HOXA genes. B, GSEA analysis showing significant enrichment of genes at the chromosome $7 \mathrm{p} 15$ locus (enrichment score $=0.68$, nominal $P<0.001$, FDR $q=0.930$ ), and of HOXA genes in particular (enrichment score $=0.90$, nominal $P<0.001$, FDR $q<0.001$ ), upregulated in short-term survivors. C, Kaplan-Meier plot showing a significantly shorter survival of patients with high levels of HOXA9/HOXA10 gene expression $(P=0.0453$, log-rank test).

significantly reduced overall survival of pediatric high-grade glioma patients with high $H O X A 9 / H O X A 10$ expression (logrank test, $P=0.0453$; Fig. $6 \mathrm{C}$ ) independent of the WHO grade of the tumor $(P=0.635$, Fisher's exact test).

\section{Discussion}

Promoter methylation of the MGMT gene is generally accepted as the major determinant of sensitivity to the alkylating agent temozolomide in glioblastoma cells, and as such has major significance in the treatment of these patients. We identified the pediatric glioblastoma cell line KNS42 to be resistant to temozolomide in vitro despite an absence of MGMT expression, a competent MMR system, and an intact double-strand break repair pathway. Clues as to the mechanism of resistance in these cells may help in identifying factors that contribute to childhood glioblastoma patients who remain refractory to temozolomide treatment.
Gene expression profiling of a panel of pediatric and adult glioma cell lines highlighted coordinated expression of numerous $H O X$ genes in the resistant cell lines, most especially KNS42, and provided in vitro model system evidence in support of data from temozolomide-treated adult glioblastoma patients (26). Using a similar expression profiling and GSEA approach, Murat and coworkers identified a $H O X$-dominated gene cluster as an independent predictive factor of resistance. Integrating the core gene lists of the present study to that dataset highlights $H O X A 9$ and $H O X A 10$ as the key effectors in both systems. This converges with a recent study identifying the HOXA cluster, and HOXA9 in particular, to be independent negative prognostic markers in adult glioblastoma (27). Herein we provide evidence for an additional prognostic role in pediatric high-grade glioma.

$H O X$ genes are essential in axis determination during embryonic development and are known to be involved in cancer, including glioblastomas $(31,32)$; it is not immediately apparent, however, what role they may play in resistance to 
alkylating agents. Costa and colleagues propose that HOXA9 exerts antiapoptotic and proproliferative effects after upregulation via an epigenetic mechanism controlled by PI3K and independent of mTOR (27). Our findings confirm this observation, and add to previous evidence showing the synergistic interactions of temozolomide and PI3K pathway inhibitors in in vitro and in vivo models of adult glioblastoma (33); this combination may therefore also be beneficial in glioblastoma patients with a MGMT-independent $H O X$ gene signatureassociated mechanism of resistance to temozolomide.

Murat and colleagues suggested strong HOXA10 expression in glioblastoma-derived neurospheres to be in line with a role of $H O X$ genes in the glioma stem-like cell compartment, and showed that the resistance signature as a whole is evocative of self-renewal (26). Of note is the presence of a high proportion (17.0\%) of CD133-positive cells present in our KNS42 cell monolayer cultures, with additional expression of other stem cell markers in coordination with the $H O X$ gene signature. Such a $H O X /$ stem cell signature was also found to be tightly regulated in an analysis of TCGA glioblastoma expression data (21). The pediatric glioblastoma cell line KNS42 may be an excellent experimental model for investigating such interactions with MGMT-independent treatment failure.

We also found a remarkable link between the $\mathrm{HOX} /$ stem cell signature and coordinated overexpression of genes within the CDK4 amplicon at 12q13-q14 in glioblastoma patient samples. This association was also present, and correlated with poor response to temozolomide chemoradiotherapy, in the Murat and colleagues dataset (26). Here we provide a possible mechanism for this coexpression of $H O X$ and 12q13-q14 genes in the form of overexpression of the Akt enhancer PIKE (CENTG1, AGAP2), also present at very high levels in the unamplified KNS42 cells, which may drive the changes in H3K27 methylation of the HOXA cluster mediated via PI3K pathway signaling (27).

\section{References}

1. Mrugala MM, Chamberlain MC. Mechanisms of disease: temozolomide and glioblastoma-look to the future. Nat Clin Pract Oncol 2008; 5:476-86.

2. Stupp R, Mason WP, van den Bent MJ, et al. Radiotherapy plus concomitant and adjuvant temozolomide for glioblastoma. N Engl J Med 2005;352:987-96.

3. Newlands ES, Blackledge GR, Slack JA, et al. Phase I trial of temozolomide (CCRG 81045: M\&B 39831: NSC 362856). Br J Cancer 1992;65:287-91.

4. Hegi ME, Diserens AC, Gorlia T, et al. MGMT gene silencing and benefit from temozolomide in glioblastoma. N Engl J Med 2005;352: 997-1003.

5. Esteller M, Garcia-Foncillas J, Andion E, et al. Inactivation of the DNA-repair gene MGMT and the clinical response of gliomas to alkylating agents. N Engl J Med 2000;343:1350-4.

6. Middlemas DS, Stewart CF, Kirstein MN, et al. Biochemical correlates of temozolomide sensitivity in pediatric solid tumor xenograft models. Clin Cancer Res 2000;6:998-1007.

7. Silber JR, Bobola MS, Blank A, et al. The apurinic/apyrimidinic endonuclease activity of Ape1/Ref-1 contributes to human glioma cell resistance to alkylating agents and is elevated by oxidative stress. Clin Cancer Res 2002;8:3008-18.
It is apparent that a variety of processes, not all involving repair or tolerance of alkyl lesions, may promote alkylator resistance (23). Along with providing therapeutic guidance for those patients whose tumors are intrinsically resistant to treatment, characterization of additional determinants of resistance is necessary to develop new targets for therapy in tumors that acquire resistance to temozolomide in vivo in the presence of hypermethylated MGMT.

\section{Disclosure of Potential Conflicts of Interest}

N. Gaspar, L. Marshall, L. Perryman, D.A. Bax, S.E. Little, M. Viana-Pereira, S.Y. Sharp, A.D.J. Pearson, P. Workman, and C. Jones are or were employees of The Institute of Cancer Research, which has a commercial interest in the development of PI3K inhibitors and operates a rewards-to-inventors scheme. P. Workman and his team have been involved in a commercial collaboration with Yamanouchi (now Astellas Pharma) and with Piramed Pharma, and intellectual property arising from the program has been licensed to Genentech. P. Workman was a founder of, consultant to, Scientific Advisory Board member of, and stockholder in Piramed Pharma, which was acquired by Roche.

\section{Acknowledgments}

We thank Dr. Daphne Haas-Kogan (University of California, San Francisco) and Dr. Michael Bobola (University of Washington) for provision of the pediatric glioma cell lines, and Dr. Michael Hubank (Institute of Child Health, University College London) for assistance with the expression profiling.

\section{Grant Support}

Cancer Research UK (C1178/A10294, C309/A2187, C309/A8274), the Oak Foundation (L. Marshall), and La Fondation de France (N. Gaspar). We acknowledge NHS funding to the NIHR Biomedical Research Centre. P. Workman is a Cancer Research UK Life Fellow.

The costs of publication of this article were defrayed in part by the payment of page charges. This article must therefore be hereby marked advertisement in accordance with 18 U.S.C. Section 1734 solely to indicate this fact.

Received 04/09/2010; revised 09/14/2010; accepted 09/17/2010; published OnlineFirst 10/08/2010.

8. Cheng CL, Johnson SP, Keir ST, et al. Poly(ADP-ribose) polymerase1 inhibition reverses temozolomide resistance in a DNA mismatch repair-deficient malignant glioma xenograft. Mol Cancer Ther 2005; 4:1364-8.

9. Pollack IF, Hamilton RL, Sobol RW, et al. O6-methylguanine-DNA methyltransferase expression strongly correlates with outcome in childhood malignant gliomas: results from the CCG-945 Cohort. $\mathrm{J}$ Clin Oncol 2006;24:3431-7.

10. Broniscer A, Chintagumpala M, Fouladi M, et al. Temozolomide after radiotherapy for newly diagnosed high-grade glioma and unfavorable low-grade glioma in children. J Neurooncol 2006;76:313-9.

11. Nicholson HS, Kretschmar CS, Krailo M, et al. Phase 2 study of temozolomide in children and adolescents with recurrent central nervous system tumors: a report from the Children's Oncology Group. Cancer 2007;110:1542-50.

12. Ruggiero A, Cefalo G, Garre ML, et al. Phase II trial of temozolomide in children with recurrent high-grade glioma. J Neurooncol 2006;77: 89-94.

13. Hargrave DR, Zacharoulis S. Pediatric CNS tumors: current treatment and future directions. Expert Rev Neurother 2007;7:1029-42.

14. Lashford LS, Thiesse $P$, Jouvet $A$, et al. Temozolomide in malignant gliomas of childhood: a United Kingdom Children's Cancer Study 
Group and French Society for Pediatric Oncology Intergroup Study. $\mathrm{J}$ Clin Oncol 2002;20:4684-91.

15. Bax DA, Little SE, Gaspar N, et al. Molecular and phenotypic characterisation of paediatric glioma cell lines as models for preclinical drug development. PLoS ONE 2009;4:e5209.

16. Skehan $P$, Storeng $R$, Scudiero $D$, et al. New colorimetric cytotoxicity assay for anticancer-drug screening. J Natl Cancer Inst 1990;82:1107-12.

17. Cory AH, Owen TC, Barltrop JA, Cory JG. Use of an aqueous soluble tetrazolium/formazan assay for cell growth assays in culture. Cancer Commun 1991;3:207-12.

18. Chou TC, Talaly P. A simple generalized equation for the analysis of multiple inhibitions of Michaelis-Menten kinetic systems. J Biol Chem 1977;252:6438-42.

19. Esteller M, Hamilton SR, Burger PC, Baylin SB, Herman JG. Inactivation of the DNA repair gene O6-methylguanine-DNA methyltransferase by promoter hypermethylation is a common event in primary human neoplasia. Cancer Res 1999;59:793-7.

20. Nygren AO, Ameziane N, Duarte HM, et al. Methylation-specific MLPA (MS-MLPA): simultaneous detection of CPG methylation and copy number changes of up to 40 sequences. Nucleic Acids Res 2005;33:e128.

21. McLendon R, Friedman A, Bigner $D$, et al. Comprehensive genomic characterization defines human glioblastoma genes and core pathways. Nature 2008;455:1061-8.

22. Paugh BS, Qu C, Jones $C$, et al. Integrated molecular profiling of pediatric high grade gliomas reveals key differences with the adult disease. J Clin Oncol 2010;28:3061-8.

23. Bobola MS, Silber JR, Ellenbogen RG, Geyer JR, Blank A, Goff RD. O6-methylguanine-DNA methyltransferase, O6-benzylguanine, and resistance to clinical alkylators in pediatric primary brain tumor cell lines. Clin Cancer Res 2005;11:2747-55.
24. Workman $P$, van Montfort RL. Unveiling the secrets of the ancestral PI3 kinase Vps34. Cancer Cell 2010;17:421-3.

25. Subramanian A, Tamayo P, Mootha VK, et al. Gene set enrichment analysis: a knowledge-based approach for interpreting genomewide expression profiles. Proc Natl Acad Sci U S A 2005;102: $15545-50$.

26. Murat A, Migliavacca E, Gorlia T, et al. Stem cell-related "self-renewal" signature and high epidermal growth factor receptor expression associated with resistance to concomitant chemoradiotherapy in glioblastoma. J Clin Oncol 2008;26:3015-24.

27. Costa BM, Smith JS, Chen $\mathrm{Y}$, et al. Reversing HOXA9 oncogene activation by PI3K inhibition: epigenetic mechanism and prognostic significance in human glioblastoma. Cancer Res 2010;70: 453-62.

28. Hayakawa $\mathrm{M}$, Kaizawa $\mathrm{H}$, Moritomo $\mathrm{H}$, et al. Synthesis and biological evaluation of pyrido[ $\left[3^{\prime}, 2^{\prime}: 4,5\right]$ furo[3,2-d]pyrimidine derivatives as novel PI3 kinase p110alpha inhibitors. Bioorg Med Chem Lett 2007;17:2438-42.

29. Raynaud FI, Eccles S, Clarke PA, et al. Pharmacologic characterization of a potent inhibitor of class I phosphatidylinositide 3-kinases. Cancer Res 2007;67:5840-50.

30. Workman P, Collins I. Probing the probes: fitness factors for small molecule tools. Chem Biol 2010;17:561-77.

31. Abate-Shen C. Deregulated homeobox gene expression in cancer: cause or consequence?. Nat Rev Cancer 2002;2:777-85.

32. Abdel-Fattah R, Xiao A, Bomgardner D, Pease CS, Lopes MB, Hussaini IM. Differential expression of HOX genes in neoplastic and non-neoplastic human astrocytes. J Pathol 2006;209:15-24.

33. Guillard S, Clarke PA, Te Poele R, et al. Molecular pharmacology of phosphatidylinositol 3-kinase inhibition in human glioma. Cell Cycle 2009;8:443-53. 


\section{MGMT-Independent Temozolomide Resistance in Pediatric Glioblastoma Cells Associated with a PI3-Kinase-Mediated HOX/Stem Cell Gene Signature}

Nathalie Gaspar, Lynley Marshall, Lara Perryman, et al.

Cancer Res 2010;70:9243-9252. Published OnlineFirst October 8, 2010.

Updated version Access the most recent version of this article at: doi:10.1158/0008-5472.CAN-10-1250

Cited articles This article cites 33 articles, 13 of which you can access for free at: http://cancerres.aacrjournals.org/content/70/22/9243.full\#ref-list-1

Citing articles This article has been cited by 15 HighWire-hosted articles. Access the articles at: http://cancerres.aacrjournals.org/content/70/22/9243.full\#related-urls

E-mail alerts Sign up to receive free email-alerts related to this article or journal.

Reprints and To order reprints of this article or to subscribe to the journal, contact the AACR Publications Subscriptions Department at pubs@aacr.org.

Permissions To request permission to re-use all or part of this article, use this link http://cancerres.aacrjournals.org/content/70/22/9243.

Click on "Request Permissions" which will take you to the Copyright Clearance Center's (CCC) Rightslink site. 